DRAFT VERSION SEPTEMBER 28, 2018

Preprint typeset using LTEX style emulateapj v. 12/16/11

\title{
NUSTAR VIEW OF THE CENTRAL REGION OF THE PERSEUS CLUSTER
}

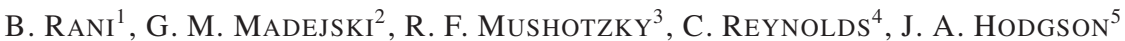 \\ ${ }^{1}$ NASA Goddard Space Flight Center, Greenbelt, MD, 20771, USA \\ ${ }^{2}$ Kavli Institute for Particle Astrophysics and Cosmology, SLAC and Stanford University, Stanford, CA 94305 \\ 3 Department of Astronomy, University of Maryland, College Park, MD 20742, USA \\ ${ }^{4}$ Institute of Astronomy, University of Cambridge, Madingley Road, Cambridge, CB3 OHA, UK and \\ ${ }^{5}$ Korea Astronomy and Space Science Institute, 776 Daedeokdae-ro, Yuseong-gu, Daejeon, 30455, Korea \\ Draft version September 28, 2018
}

\begin{abstract}
Located at the center of the Perseus cluster, 3C 84 is an extremely bright and nearby radio galaxy. Because of the strong diffuse thermal emission from the cluster in X-rays, the detailed properties and the origin of a power-law component from the central active galactic nucleus (AGN) remains unclear in the source. We report here the first NUSTAR observations of 3C 84. The source was observed for 24.2 and 32 ks on February 01 and 04, 2018, respectively. NUSTAR observations spectrally decompose the power-law AGN component above $10 \mathrm{keV}$. The power-law component dominates the spectrum above $20 \mathrm{keV}$ with a photon index $\sim 1.9$ and an energy flux $\mathrm{F}_{20-30} \mathrm{keV}=1.0 \times 10^{-11} \mathrm{erg} \mathrm{cm}^{-2} \mathrm{~s}^{-1}$, corresponding to an isotropic luminosity, $L_{20-30 \mathrm{keV}}=$ $7.4 \times 10^{42} \mathrm{erg} \mathrm{s}^{-1}$. We discuss possible emitting sites for the power-law component. The expected thermal emission from the accretion disk is not hot enough to account for the hard X-rays detected from the source. Similar X-ray and $\gamma$-ray photon indices and long-term flux variations, the absence of cutoff energy in the hard X-ray spectrum of the source, correlated hard X-ray flux and hardness ratio variations, and the similarity of optical-X-ray slope to blazar rather than Seyfert galaxies supports the hard X-ray power-law component originating from the jet.
\end{abstract}

Subject headings: galaxies: active - quasars: individual: 3C 84 - X-rays

\section{INTRODUCTION}

The Perseus cluster of galaxies is one of the X-ray brightest and nearest clusters in the sky. At the center of the Perseus cluster, 3 C 84 is a nearby $(\mathrm{z}=0.0172)$ radio loud active galactic nucleus (AGN) with a black hole mass $\sim(3.4-$ 8.0) $\times 10^{8} \mathrm{M}_{\odot}($ Wilman et al. 2005; Scharwächter et al. 2013) and a relatively low Eddington ratio, $L_{B} / L_{E d d} \sim 3 \times 10^{-4}$ (Sikora et al. 2007). The source is classified as a Seyfert Type 1.5 galaxy (Ho et al. 1997). Being at the center of a bright cluster, the X-ray properties of 3C 84 have been extensively studied. Fabian et al. (2015) compared the longterm X-ray variations with $90 \mathrm{GHz}$ radio flux and found similar variations in the two. XMM-Newton observations taken in 2001 suggest the presence of a narrow $\mathrm{Fe}-\mathrm{K} \alpha$ line in the spectrum (Churazov et al. 2003). The recent observations by the Hitomi/Soft X-ray telescope (SXS) confirm the detection of the $6.4 \mathrm{keV} \mathrm{Fe}-\mathrm{K} \alpha$ line at the $5.4 \sigma$ level, and suggest that the line originates within $\sim 1.6 \mathrm{kpc}$ of the $3 \mathrm{C} 84$ core (Hitomi Collaboration et al. 2018).

The source has been detected at $\mathrm{GeV}$ energies since the beginning of Fermi Large Area Telescope (LAT) observations (Abdo et al. 2009). Being relatively inactive throughout the 1990s, there has been an increase in $\gamma$-ray flux and cm-radio activity, which appears to have begun in 2005 (Abdo et al. 2009; Nagai et al. 2010; Suzuki et al. 2012). From December 2016 to early January 2017, the source was detected at $\mathrm{TeV}$ energies (Mukheriee \& VERITAS Collaboration 2017; Mirzoyan 2017; MAGIC collaboration et al. 2018). An increase in the radio, soft X-ray and $\gamma$-ray flux was also detected during this flaring activity period (Hodgson et al. 2018; Fukazawa et al. 2018).

NPP Fellow

bindu.rani@nasa.gov

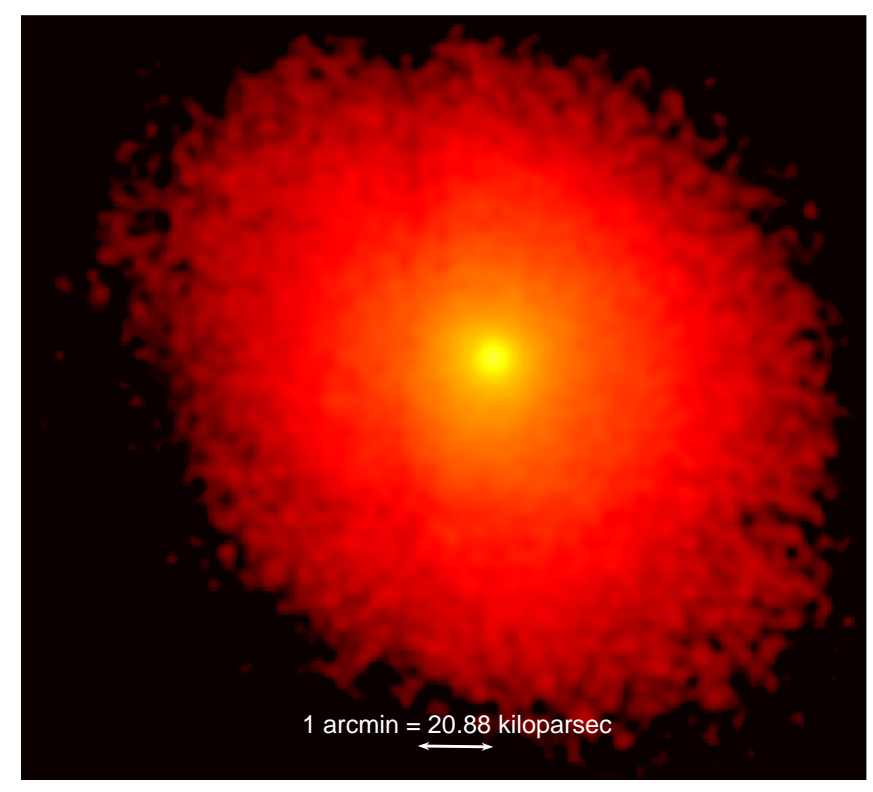

FIG. 1.- NUSTAR image of the Perseus cluster in the 3-70 KeV Kev band taken on Feb. 04, 2017. The bright emission at the center of the image is $3 \mathrm{C}$ 84

The X-ray emission of the source is strongly dominated by the thermal diffuse radiation from the cluster and a power-law component associated with the central AGN (Hitomi Collaboration et al. 2018; Ajello et al. 2009). However the physical origin this power-law component, e.g., whether it is more similar to the jet-like origin of the X-ray continuum in blazars or the thermal Comptonization component in Seyfert galaxies, is not clear. Similarities in the $\mathrm{X}$-ray and radio/ $\gamma$-ray flux variations (Fabian et al. 2015; 
Fukazawa et al.2018) clearly hint in favor of the presence of a jet-based emission component. We present here the first $\mathrm{NuS}$ $T A R$ observations of the Perseus cluster, which spectrally decompose the power-law emission component from the central AGN, and present the Swift-BAT data revealing variability of the hard X-ray flux. This paper is structured as follows. Observations and data reduction are described in Sect. 2. Analysis and results are given in Sect. 3, and Sect. 4 and 5 presents the discussion and conclusions, respectively.

\section{OBSERVATIONS AND DATA REDUCTION}

At the end of 2016, 3C 84 went through an extreme flaring activity at GeV/TeV energies (Mirzoyan 2017; Hodgson et al. 2018). To follow up the flaring activity at hard X-rays, we requested two NUSTAR (Harrison et al. 2013) observations of the source. NUSTAR observations were taken in February 2017 (observations IDs : 90202046002, 90202046004). The pointing on February 01, 2017, resulted in 24.2 ks of observing time, while on February 04, 2017, 32 ks were obtained. We also use the archival 105 months of Swift/BAT data, obtained from Oh et al. (2018); we detail the spectral fitting of those data in Section 4.2

Data were analyzed using the NuSTAR Data Analysis Software (NuSTARDAS) package v.1.3.1. After processing the raw data via nupipeline, we used nuproducts to extract higher level products, i.e. source spectra and light curves. A hard X-ray image of the source can be seen in Fig. 1. Since the Perseus cluster is a spatially extended source, we used different approaches to extract the flux and spectrum of the central AGN 3C 84. Data for the central AGN component were extracted from a region of 30 " radius, centered on the position of 3C $84(\mathrm{RA}=3: 19: 48.38, \mathrm{Dec} .=+41: 30: 42.10)$. Background was extracted from a 30 " radius region roughly 180 " SW of the source location. We also cross-check the results by selecting an annular background region centered at the source position with an inner radius of 200" and an outer radius of 230 ". We binned the spectra in order to have at least 30 counts per rebinned channel. For spectral fitting, we considered the channels corresponding nominally to the 3-70 keV energy range, where the source was robustly detected. In either case, we treat the thermal cluster emission as a background, and do not care about the residual thermal cluster flux in the source extraction region, only about the hard X-ray emission dominating at higher energies. Our implicit assumption is that the residual cluster emission at the $3 \mathrm{C} 84$ extraction region and at the region used by us as background, have roughly the same temperature (Schmidt et al. 2002).

\section{ANALYSIS AND RESULTS}

\subsection{Nustar data}

We used XSPEC V15.4.0 to model the source spectrum. In addition to an absorption model (wabs), which describes the absorption in the AGN by cold absorbing material in our Galaxy, we used a combined power-law, $A(E)=K E^{-\alpha}$, and an emission spectrum from hot diffuse gas, apec, to fit the source spectrum, i.e. wabs * (apec + powerlaw). In wabs, we fixed the column density $(n H)$ to the Galactic value of $1.4 \times 10^{21} \mathrm{~cm}^{-2}$. The redshift $(z)$ of the source is fixed to 0.017 . The plasma temperature $(k T)$, metal abundance (abund), and apec normalization (Norm) are kept free while fitting the spectrum. Both the photon index $(\alpha)$ and normalization $(K)$ of the power - law are also set free. The model fit parameters and the reduced $\chi^{2}$ values along
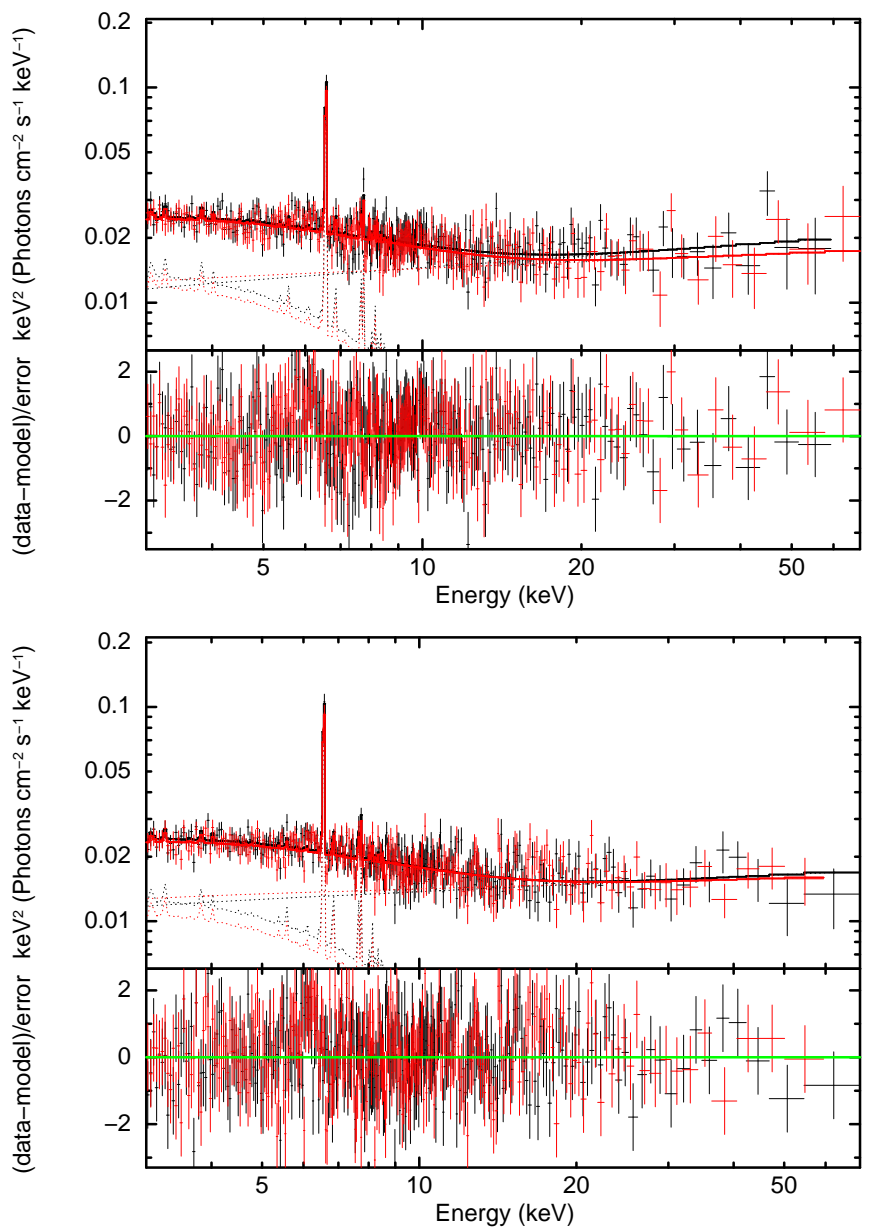

FIG. 2.- Fitted NUSTAR spectrum of 3C 84 - data taken on Feb. 01, 2017 (middle) and Feb. 04, 2017 (bottom). Plus symbols are the observed data while the lines represent the fitted models.

with the degrees of freedom and the corresponding probability are given in Table 1 As the statistics suggest, this simple wabs * (apec + powerlaw $)$ model well describes the source spectrum over the energy range form 3.0 to $70.0 \mathrm{keV}$.

Motivated by the detection by the Hitomi satellite of the Fe $\mathrm{K} \alpha$ line emission from the AGN (Hitomi Collaboration et al. 2018), we searched for a presence of such a line in the NuSTAR data. The flux of the sum of the two components of the line measured by Hitomi is modest, at $\sim 6 \times 10^{-6}$ photons $\mathrm{cm}^{-2} \mathrm{~s}^{-1}$. We added a narrow Gaussian emission component to our combined apec + powerlaw model, and note a significant improvement in $\chi^{2}$ (with $\Delta \chi^{2}$ of 10 for either observation). We note that the best-fit of the line flux is significantly greater than that measured by Hitomi (by about a factor of 5 , at $\sim 30 \times 10^{-6}$ photons $\left.\mathrm{cm}^{-2} \mathrm{~s}^{-1}\right)$. However, the fitted energy (as observed) is not well determined (values between 6.0 and $6.4 \mathrm{keV}$ are allowed). In conclusion, we consider as a possibility to account for this finding the fact that the weak Fe-K line is sitting on the wing of the very strong (but intrinsically narrow) $6.7 \mathrm{keV}$ (rest) emission line from the hot cluster gas, and our detection is an artifact of slight miscalibration of NuSTAR's spectral response (specifically, the low-energy wing) to a narrow line.

A significant dominance of the thermal diffuse emission (see Fig. 2) in the source spectrum is seen up to $\sim 10 \mathrm{keV}$. The power-law AGN component clearly takes over above $10 \mathrm{keV}$. Most of the radiation we see above $20 \mathrm{keV}$ is contributed 
TABLE 1

PARAMETERS OF THE SPECTRAL FITS

\begin{tabular}{|c|c|c|c|c|c|}
\hline \multirow{2}{*}{$\begin{array}{l}\text { Model } \\
\text { component }\end{array}$} & \multirow[t]{2}{*}{ Parameter } & \multicolumn{2}{|c|}{ Period A } & \multicolumn{2}{|c|}{ Period B } \\
\hline & & FPMA & FPMB & FPMA & FPMB \\
\hline wabs & $\mathrm{nH}$ (column density in units of $10^{22} \mathrm{~cm}^{-2}$ ) & 0.14 (frozen) & & & \\
\hline \multirow[t]{4}{*}{ apec } & $\mathrm{KT}$ (plasma temperature in $\mathrm{keV}$ ) & $3.47_{+0.35}^{-0.42}$ & - & $3.70_{+0.34}^{-0.29}$ & - \\
\hline & Abun (metal abundances) & $0.39_{+0.19}^{-0.09}$ & - & $0.39_{+0.16}^{-0.11}$ & - \\
\hline & $\mathrm{z}$ (redshift) & 0.017 (frozen) & & & \\
\hline & Norm (apec normalization) & $0.044_{+0.012}^{-0.011}$ & $0.039_{+0.010}^{-0.011}$ & $0.039_{+0.008}^{-0.007}$ & $0.034_{+0.008}^{-0.008}$ \\
\hline \multirow[t]{2}{*}{ power - law } & $\alpha$ (power-law index) & $1.85_{+0.12}^{-0.14}$ & - & $1.90_{+0.11}^{-0.13}$ & - \\
\hline & K (power-law normalization) & $0.00974_{+0.0049}^{-0.0033}$ & $0.0114_{+0.0048}^{-0.0032}$ & $0.0106_{+0.0037}^{-0.0036}$ & $0.0120_{+0.0037}^{-0.0035}$ \\
\hline$\chi^{2} /$ dof (prob.) & reduced $\chi^{2} /$ degrees of freedom (a corresponding probability) & $0.89 / 454(0.96)$ & - & $1.03 / 564(0.28)$ & - \\
\hline $\mathrm{F}_{20-30 \mathrm{keV}}$ & AGN flux in units of $10^{-11} \mathrm{erg} \mathrm{cm}^{-2} \mathrm{~s}^{-1}$ & $1.01_{+0.01}^{-0.05}$ & $0.99_{+0.01}^{-0.03}$ & $0.99_{+0.19}^{-0.37}$ & $0.98_{+0.01}^{-0.20}$ \\
\hline \multirow[t]{2}{*}{$\mathrm{F}_{2-10 \mathrm{keV}}$} & AGN flux in units of $10^{-11} \mathrm{erg} \mathrm{cm}^{-2} \mathrm{~s}^{-1}$ & $3.36_{+0.03}^{-0.03}$ & $3.5_{+0.08}^{-0.08}$ & $3.20_{+0.05}^{-0.04}$ & $3.40_{+0.06}^{-0.05}$ \\
\hline & COMBINED NUSTAR AND BAT SPECTRAL FITS & & & & \\
\hline Model1: & Parameter & NuSTAR data & BAT data & & \\
\hline apec & $\mathrm{kT}(\mathrm{keV})$ & $3.56_{+0.18}^{-0.25}$ & $4.02_{+0.24}^{-0.31}$ & & \\
\hline apec & norm & $0.031_{+0.002}^{-0.002}$ & $0.82_{+0.13}^{-0.13}$ & & \\
\hline powerlaw & PhoIndex & $1.96_{+0.09}^{-0.04}$ & - & & \\
\hline powerlaw & norm & $0.013_{+0.002}^{-0.002}$ & $0.008_{+0.003}^{-0.002}$ & & \\
\hline$\chi^{2} /$ dof (prob.) & & $578 / 577(0.20)$ & - & & \\
\hline Model2: & Parameter & NuSTAR data & BAT data & & \\
\hline apec & $\mathrm{kT}(\mathrm{keV})$ & $3.45_{+0.52}^{-0.33}$ & $3.84_{+0.85}^{-0.73}$ & & \\
\hline apec & norm & $0.031_{+0.003}^{-0.003}$ & $\begin{array}{l}97_{+80}^{-70} \\
0\end{array}$ & & \\
\hline cutoffpl & PhoIndex & $\begin{array}{l}1.94_{+0.03}^{-0.03} \\
\end{array}$ & - & & \\
\hline cutoffpl & norm & $0.013_{+0.002}^{-0.002}$ & $0.008_{+0.003}^{-0.004}$ & & \\
\hline cutoffpl & HighECut (keV) & $>100$ & $>100$ & & \\
\hline$\chi^{2} /$ dof (prob.) & & $577 / 558(0.21)$ & - & & \\
\hline Model3: & Parameter & NUSTAR data & BAT data & & \\
\hline apec & $\mathrm{kT}(\mathrm{keV})$ & $3.93_{+0.45}^{-0.33}$ & $4.95_{+0.79}^{-0.94}$ & & \\
\hline apec & norm & $0.028_{+0.003}^{-0.002}$ & $16_{+13}^{-17}$ & & \\
\hline pexrav & PhoIndex & $2.08_{+0.05}^{-0.21}$ & - & & \\
\hline pexrav & foldE (keV) & $>100$ & $>100$ & & \\
\hline pexrav & rel_refl & $0.50_{+0.45}^{-0.40}$ & - & & \\
\hline pexrav & Redshift & 0.017 (frozen) & - & & \\
\hline pexrav & abund & 1.0 (frozen) & - & & \\
\hline pexrav & Fe_abund & 1.0 frozen) & - & & \\
\hline pexrav & cosIncl & 0.45 (frozen) & - & & \\
\hline pexrav & norm & $0.016_{+0.003}^{-0.003}$ & $0.011_{+0.005}^{-0.004}$ & & \\
\hline$\chi^{2} /$ dof (prob.) & & $575 / 558(0.22)$ & - & & \\
\hline
\end{tabular}

The following parameters are Fixed in Model1, Model2, and Model3: $\mathrm{nH} / \mathrm{wabs}\left(10^{20} \mathrm{~cm}^{-2}\right)=0.14$, Abundanc/apec $=0.50$, Redshift $=0.017$. We used the AtomDB (version 3.0.9) and the default abundance table is from Anders \& Grevesse (1989).

Note: The uncertainties on the parameters are $90 \%$ confidence limits.

by the central AGN, 3C 84. In both epochs of our observations, the photon index is determined to be $1.8-1.9$, which is quite similar to the $\gamma$-ray photon index during that period, $\Gamma_{E>100 \mathrm{MeV}} \sim 2.0$ (Hodgson et al. 2018). The $20-30 \mathrm{keV}$ energy flux range is $0.9-1.1 \times 10^{-11} \mathrm{erg} \mathrm{cm}^{-2} \mathrm{~s}^{-1}$, corresponding to an apparent isotropic X-ray luminosity, $L_{20-30 \mathrm{keV}}$, of $(7.35 \pm 0.66) \times 10^{42} \mathrm{erg} \mathrm{s}^{-1}$. We do not observe any variation in the photon index or flux across the 4 day gap of the two NUSTAR observations (see Table 1).

The estimated soft X-ray, 2-10 keV, flux of the central region (including both the AGN and the residual cluster emission in the 30 arcsec radius extraction region) is $\sim 6 \times 10^{-11} \mathrm{erg} \mathrm{cm}{ }^{-2} \mathrm{~s}^{-1}$. Soft X-ray flux of the AGN component is $\sim 3.3 \times 10^{-11} \mathrm{erg} \mathrm{cm}-2 \mathrm{~s}^{-1}\left(L_{2-10 \mathrm{keV}}=\right.$ $\sim 2.3 \times 10^{43} \mathrm{erg} \mathrm{s}^{-1}$ ), which is similar to the AGN continuum flux, $\mathrm{F}_{2-10 \mathrm{keV}} \sim(1.96-4.36) \times 10^{-11} \mathrm{erg} \mathrm{cm}^{-2} \mathrm{~s}^{-1}$, measured by the Hitomi/SXS observations in February, 2016 (Hitomi Collaboration et al. 2018). The photon index is also similar for the two observations. Hitomi/SXS measured the power-law index in the range 1.45-2.06, and for the NuSTAR observations, it is $\sim 1.9$. The improved constraints relative to Hitomi result from NuSTAR's superior hard X-ray coverage (with sensitivity out to $70 \mathrm{keV}$ ) and spatial resolution.

\subsection{Swift/BAT data}

We used the archival Swift/BAT data over the first 105 months of observations from December 2004 to August 2013 (Oh et al. 2018) to investigate the hard X-ray spectral properties of the source. The detailed analysis of the BAT data can be found in $\mathrm{Oh}$ et al. (2018). We performed a combined fit of the NUSTAR and BAT data, which we discuss in Section 4.2 Note that the NUSTAR and BAT observations are not close in time and the BAT data are averaged over 9 years.

\section{DISCUSSION}

Given its high sensitivity and angular resolution at hard Xrays, NUSTAR observations spectrally decompose the power- 
law component in the $3 \mathrm{C} 84$ spectrum. The power-law component of the spectrum could either be from the immediate vicinity, i.e. disk/corona, of the central black hole or from the jet. In the following sub-sections we discuss these possibilities in detail.

\subsection{Accretion disk}

For a $3-8 \times 10^{8} \quad \mathrm{M}_{\odot}$ black hole accreting at $10 \%$ of its Eddington rate, the peak temperature at $1 R_{S}$ is $\approx 2.5 \times 10^{5} \mathrm{~K}$, corresponding to $5.2 \times 10^{15} \mathrm{~Hz}$, which falls in the ultraviolet (UV) band. Of course for $3 \mathrm{C} \mathrm{84,} \mathrm{the} \mathrm{accretion}$ rate is way below $10 \%$ and $T \propto\left(\dot{L}_{E d d}\right)^{1 / 4}$; so, the disk would be even cooler. Moreover, the thermal radiation from the disk does not follow a power law.

\subsection{Corona}

Inverse-Compton scattering of photons from the accretion disk by the corona could emit in hard X-rays Reis \& Millen 2013; Fabian et al. 2015). For radiatively compact emission regions (like corona, $\sim 2-30 R_{q}$, Fabian et al. 2009; Wilkins \& Fabian 2011; Sanfrutos et al. 2013), pair production naturally occurs. At extremely high temperature, pair production can be a runway process, limiting any further rise in the temperature and a producing a cutoff in the spectrum, which for Seyfert galaxies is in the 50-200 KeV range (Fabian et al. 2015). However at low mass accretion rates ( $L \leq 10^{-4} L_{E d d}$ ) the compactness of the corona will be low and so very high temperatures can be achieved before the pairproduction condition is met.

In the $N U S T A R$ spectrum extending up to $70 \mathrm{keV}$, we do not see any hint of a cutoff in the spectrum. To further examine the possibility of a cutoff at higher energies, we performed a combined fit of the NUSTAR and Swift/BAT observations. We noticed a clear disagreement between $N U S T A R$ and BAT data between $\sim(15-40) \mathrm{keV}$, which could be anticipated as the data are not simultaneous. Moreover, the BAT spectrum is extracted from a larger region (19.5 arcmin) compared to $\mathrm{NuS}$ TAR and is dominated by cluster emission up to $\sim 50 \mathrm{KeV}$ (Ajello et al. 2009), which could be possibly responsible for the discrepancy between the two. Because of this reason, we ignore the BAT data below $50 \mathrm{KeV}$ in the joint NuSTAR-BAT spectral fitting.

We tried several models to fit the combined NuSTAR and BAT data. At first, we used the simple wabs * (apec + powerlaw) model (Model1) and froze the model parameters to their respective $N U S T A R$ model-fit values except the apec plasma temperature $(K T)$ and normalization and the powerlaw index and its normalization. While we present here the spectral-fit results using the NUSTAR data taken on February 04, 2017, we obtained similar results for the first NuSTAR observation. The model-fit parameters are given in Table 1 and the fitted spectrum is plotted in Fig. 3 (top). As the statistics suggest, Model1 well describes the source spectrum up to $200 \mathrm{KeV}$. Moreover, the power-law index for the combined NuSTAR-BAT data is same as the power-law index for the NUSTAR spectral fits.

We next replaced the power-law component by a cutoff power-law (cutof $f p l$ ) (Model2), and compared the test statistics of the two fits. We noticed no significant change in the $\chi^{2}$ after adding the cutoff energy. Figure 4 (top) illustrates the contour plots between the power-law photon index and cutoff energy, which constrain the cutoff energy to be above $100 \mathrm{keV}$ for values of the index between 1.7-2.1. We further tested the reflection signatures in the spectrum (Model 3
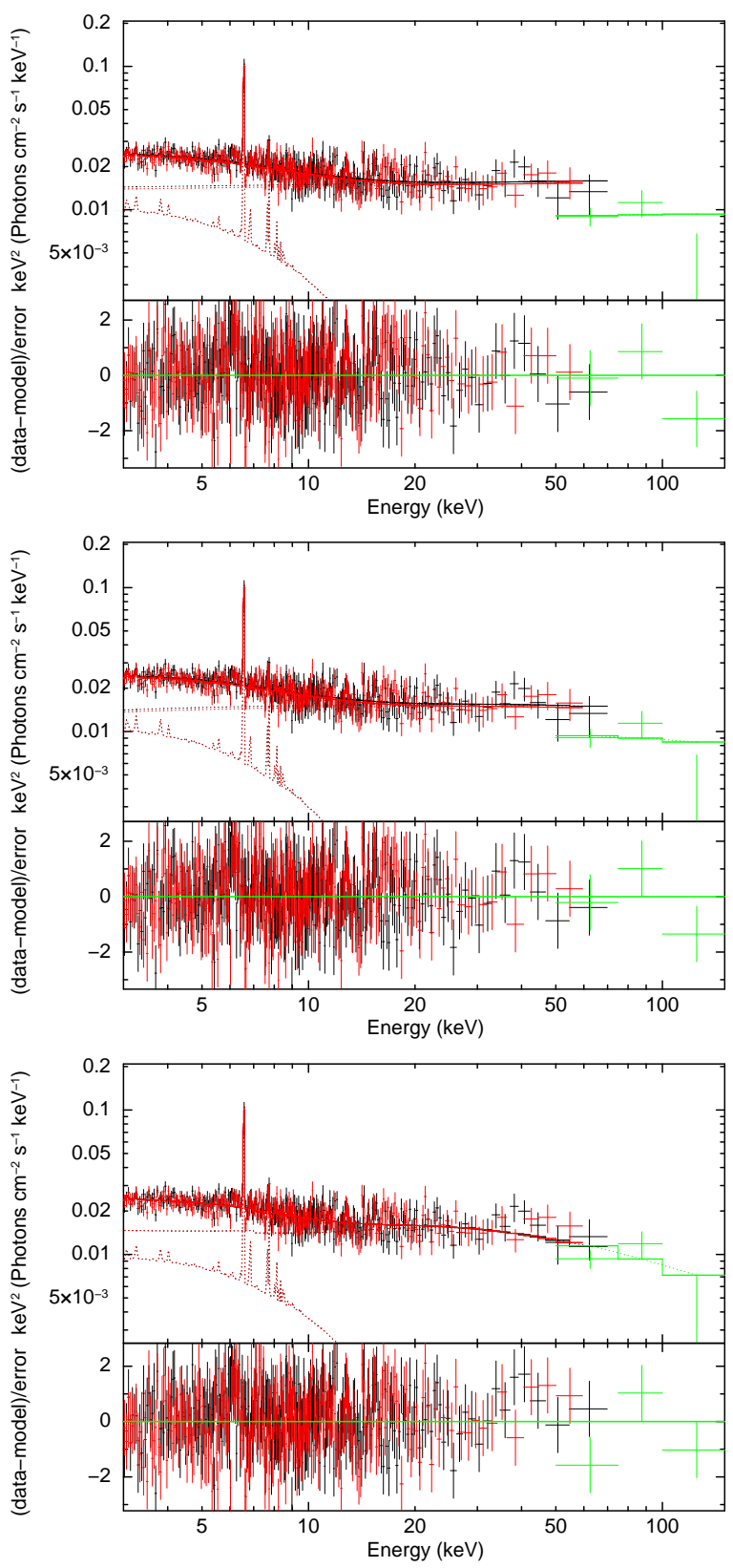

FIG. 3.- Combined NUSTAR and BAT spectrum fitted with Model1: (wabs * (apec + powerlaw), top), Model2: (wabs * (apec + cutof fpl $)$, middle), and Model3: (wabs * (apec + pexrav), bottom). The best fit parameters are listed in Table 1

Magdziarz \& Zdziarski 1995). Again no substantial improvement is noticed in the spectral fitting. The best fit parameters are listed in Table 1 and the fitted spectrum is plotted in Fig. 3. The index and cutoff energy contour plots in the reflection model are shown in Fig. 4 (bottom). The reflection model constrained the cutoff energy to be above $100 \mathrm{keV}$ for index 1.9-2.2. The absence of any significant improvement in the spectral fits by adding either cutoff energy or reflection component over a simple power-law component suggests that if there is a cutoff energy in the hard X-ray spectrum of the source, it is beyond the BAT energy range. Both spectral models constrain the cutoff energy to be above $100 \mathrm{keV}$, which argues in favor of the jet-based origin of the hard X-ray emission in the source. 

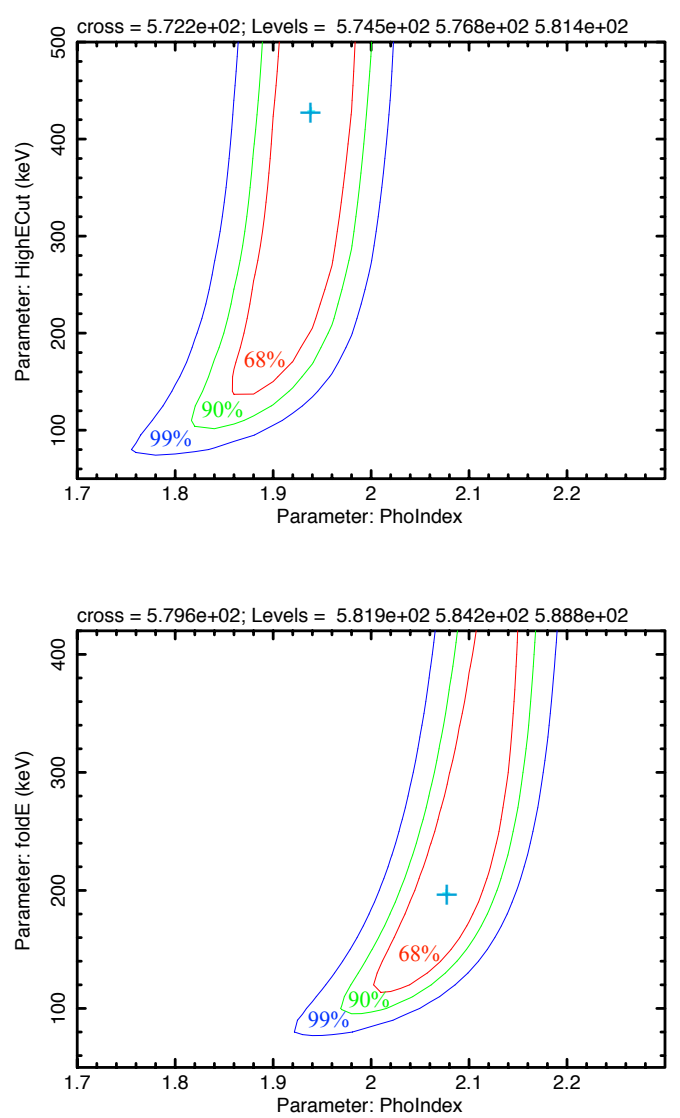

FIG. 4.- Top: Confidence contours of cutoff energy versus photon index parameters in the MODEL2 (cutoff power-law, top) and MODEL3 (reflection, bottom). The best fit parameter ("+" symbol) and 68, 90, and $99 \%$ levels are shown.

\subsection{Jet}

Since the photon index and flux behavior is quite similar in the NuSTAR X-ray and Fermi-LAT $\gamma$-ray observations, we used the NUSTAR spectral information to extrapolate and predict the $\gamma$-ray flux in the Fermi energy range using the dummyrsp routine in XSPEC. The task returns a flux value of $1.9 \times 10^{-10} \mathrm{erg} \mathrm{cm}^{-2} \mathrm{~s}^{-1}$ corresponding to an isotropic luminosity of $\sim 1.14 \times 10^{44} \mathrm{erg} \mathrm{s}^{-1}$ in the $0.3-$ $1.0 \mathrm{GeV}$ energy range. The estimated isotropic $\gamma$-ray luminosity using NUSTAR observations is similar to the observed $\gamma$-ray luminosity of the source using the Fermi-LAT, i.e. $\mathrm{L}_{\gamma-\text { ray }} \sim 10^{44} \mathrm{erg} \mathrm{s}^{-1}$ (Fukazawa et al. 2018; Hodgson et al. 2018). This suggests that the hard X-ray power-law spectrum extends up to $\gamma$ rays. If this is true, the flux variations at the two energy bands should also have similar behavior. In fact, in a recent study Fukazawa et al. (2018) found a similar variability trend in the long-term 5-10 keV X-ray and $\gamma$-ray flux variations. Moreover, the X-ray emission can be described as the low-energy tail of the inverse-Compton scattering in case of a one-zone synchrotron self-Compton model, suggesting a jetbased origin of the X-ray variations (Fukazawa et al. 2018). A significant correlation between $\gamma$-ray and radio flux variations suggests multiple high-energy emission sites located within a distance of $\sim 2.0$ parsecs from the central black hole (Hodgson et al. 2018). Correlated X-ray and $\gamma$-ray variations

\footnotetext{
${ }^{1}$ https://heasarc.gsfc.nasa.gov/xanadu/xspec/manual/node94.html
}

in the source imply the same site for the X-ray emission, i.e. the power-law component of the X-ray emission is located within 2 parsecs of the central black hole.

Another check for the jet-based origin of the hard Xray emission is the spectral slope from optical to $\mathrm{X}$-rays $\left(\alpha_{O X}\right)$. Seyfert galaxies (where most of the X-ray emission is from the immediate vicinity of the central black hole) have $\left\langle\alpha_{O X}\right\rangle \sim 1.4$ (Lusso et al. 2010). The slope could be a bit smaller for low-luminosity AGN like 3C 84 as $\alpha_{O X}$ is a function of luminosity (see Donato et al. 2001, for more details). For blazars (where X-ray emission is mostly from the jet), $\alpha_{O X}$ is $\sim 1.0-1.2$ (Donato et al. 2001). We compared the optical continuum flux at $1500 \AA$ from Evans \& Koratkar (2004) to $2-10 \mathrm{KeV} \mathrm{X}$-ray flux, and the estimated $\alpha_{O X}$ value for $3 \mathrm{C} 84$ is 1.12, which is more similar to blazars than Seyfert galaxies favoring the jet-based origin of the X-ray emission.

Using the argument that X-ray and $\gamma$-ray emission in 3C 84 are co-spatial, we derive a lower limit on the Doppler factor using (Dondi \& Ghisellini 1995),

$\delta \geq\left[3.5 \times 10^{3} \frac{(1+z)^{2 \alpha}(1+z-\sqrt{1+z})^{2} F_{x}\left(3.8 \nu \nu_{x}\right)^{\alpha}}{t_{\text {var }}}\right]^{1 /(4+2 \alpha)}$

where $\nu_{x}$ is the $\mathrm{X}$-ray frequency in $\mathrm{keV}$ and $F_{x}$ is the corresponding flux density in $\mu \mathrm{Jy}, \alpha$ is the $\mathrm{X}$-ray spectral index, $\nu$ is the $\gamma$-ray frequency, and $t_{v a r}$ is the variability timescale at high-energies (mostly used to constrain the size of the highenergy emission region). From the NuSTAR observations, we have $F_{20-30 ~ k e V}=0.16 \mu \mathrm{Jy}$ (flux density calculated from the

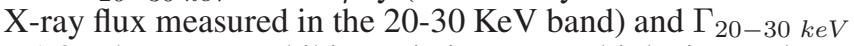
$=1.9$. The source exhibits variations on multiple timescales at $\mathrm{GeV}$ energies, i.e. the rapid flares have doubling timescales of $\sim 10 \mathrm{hr}$ (Tanada et al. 2018) while the long-duration outburst lasts for 8-12 months (250-350 days) (Hodgson et al. 2018). Using $\nu_{X-\text { ray }}=25 \mathrm{keV}, \alpha_{X-\text { ray }}=0.9\left(\Gamma_{20-30 \mathrm{keV}}-1\right)$, and $t_{\text {var }}=10 \mathrm{hr}$, we obtained $\delta_{1 \mathrm{GeV}} \geq 1.4$ and $\delta_{1 \mathrm{TeV}} \geq 3.9$. Using $t_{v a r}=300$ days, we have $\delta_{1 G e V} \geq 0.4$ and $\delta_{1 T e V} \geq 1.3$. The estimated Doppler factor values $(\bar{\delta} \geq 0.4-3.9)$ are similar to those obtained using the broadband spectral energy distribution and jet kinematics $(\delta \sim 1-3$, Fukazawa et al. 2018; Hodgson et al. 2018).

\section{CONCLUSION}

We present analysis of the NUSTAR observations of the central nucleus (3C 84) of the Perseus cluster. Because of the strong dominance of the diffuse thermal emission from the cluster, the true nature and origin of the power-law component from the central AGN remains under debate. The greater sensitivity at hard X-rays offered by NUSTAR allowed us to probe the AGN component in the source spectrum. A strong dominance of the thermal diffuse emission from the cluster is seen below $10 \mathrm{keV}$, while the power-law component takes over above $20 \mathrm{keV}$. No flux and spectral variations were observed in the two NuSTAR pointings during 4 days. The $20-30 \mathrm{keV}$ energy flux range is $\sim 1.0 \times 10^{-11} \mathrm{erg} \mathrm{cm}^{-2} \mathrm{~s}^{-1}\left(L_{20-30 \mathrm{keV}}\right.$ $=7.4 \times 10^{42} \mathrm{erg} \mathrm{s}^{-1}$ ) with a photon index of $\sim 1.9$.

We discussed the possibility of different AGN components contributing to the power-law spectrum. An accretion disk of a (3-8) $\times 10^{8} \mathrm{M}_{\odot}$ black hole does not shine in X-rays. The following arguments suggest that the hard $\mathrm{X}$-ray and $\gamma$-ray radiation is emitted by the same population of relativistic electrons from the jet: (1) X-ray and $\gamma$-ray photon indices are quite similar $(\Gamma \sim 2)$, (2) $\gamma$-ray flux is just an extension of the $\mathrm{X}$-ray flux, (3) the source exhibits similar long-term flux vari- 
ations at X-rays and $\gamma$-rays, (4) no indication of cutoff energy is seen in the combined NuSTAR and BAT spectrum, and (5) the optical-to-Xray spectral slope is more similar to that of blazars rather than Seyfert galaxies. Our study therefore supports that the hard X-ray power-law component dominating the source spectrum above $20 \mathrm{KeV}$ originates from the jet.
This research was supported by an appointment to the NASA Postdoctoral Program at the Goddard Space Flight Center, administered by Universities Space Research Association through a contract with NASA. BR thanks Erin Kara, Mihoko Yukita, Dave Thompson, Stefan Walker, Amy Lien, Hans Krimm, and Chris Shrader, for interesting discussions about AGN disk and corona.

\section{REFERENCES}

Abdo, A. A., Ackermann, M., Ajello, M., et al. 2009, ApJS, 183, 46 Ajello, M., Rebusco, P., Cappelluti, N., et al. 2009, ApJ, 690, 367

Anders, E., \& Grevesse, N. 1989, Geochim. Cosmochim. Acta, 53, 197

Churazov, E., Forman, W., Jones, C., \& Böhringer, H. 2003, ApJ, 590, 225

Donato, D., Ghisellini, G., Tagliaferri, G., \& Fossati, G. 2001, A\&A, 375, 739

Dondi, L., \& Ghisellini, G. 1995, MNRAS, 273, 583

Evans, I. N., \& Koratkar, A. P. 2004, ApJS, 150, 73

Fabian, A. C., Walker, S. A., Pinto, C., Russell, H. R., \& Edge, A. C. 2015, MNRAS, 451, 3061

Fabian, A. C., Zoghbi, A., Ross, R. R., et al. 2009, Nature, 459, 540

Fukazawa, Y., Shiki, K., Tanaka, Y., et al. 2018, ApJ, 855, 93

Harrison, F. A., Craig, W. W., Christensen, F. E., et al. 2013, ApJ, 770, 103

Hitomi Collaboration, Aharonian, F., Akamatsu, H., et al. 2018, PASJ, 70, 13

Ho, L. C., Filippenko, A. V., \& Sargent, W. L. W. 1997, ApJS, 112, 315

Hodgson, J. A., Rani, B., Lee, S.-S., et al. 2018, MNRAS, 475, 368

Lusso, E., Comastri, A., Vignali, C., et al. 2010, A\&A, 512, A34

Magdziarz, P., \& Zdziarski, A. A. 1995, MNRAS, 273, 837

MAGIC collaboration, Ansoldi, S., Antonelli, L. A., et al. 2018, ArXiv e-prints, arXiv:1806.01559
Mirzoyan, R. 2017, The Astronomer's Telegram, 9929

Mukherjee, R., \& VERITAS Collaboration. 2017, The Astronomer's Telegram, 9931

Mushotzky, R. F., Done, C., \& Pounds, K. A. 1993, ARA\&A, 31, 717

Nagai, H., Suzuki, K., Asada, K., et al. 2010, PASJ, 62, L11

Oh, K., Koss, M., Markwardt, C. B., et al. 2018, ApJS, 235, 4

Reis, R. C., \& Miller, J. M. 2013, ApJ, 769, L7

Sanfrutos, M., Miniutti, G., Agís-González, B., et al. 2013, MNRAS, 436, 1588

Scharwächter, J., McGregor, P. J., Dopita, M. A., \& Beck, T. L. 2013, MNRAS, 429, 2315

Schmidt, R. W., Fabian, A. C., \& Sanders, J. S. 2002, MNRAS, 337, 71

Sikora, M., Stawarz, Ł., \& Lasota, J.-P. 2007, ApJ, 658, 815

Suzuki, K., Nagai, H., Kino, M., et al. 2012, ApJ, 746, 140

Tanada, K., Kataoka, J., Arimoto, M., et al. 2018, ApJ, 860, 74

Wilkins, D. R., \& Fabian, A. C. 2011, MNRAS, 414, 1269

Wilman, R. J., Edge, A. C., \& Johnstone, R. M. 2005, MNRAS, 359, 755 